\title{
MODELAGEM RIGOROSA DE UM REATOR INDUSTRIAL DE HEXAMINA
}

\author{
R. M. DOURADO ${ }^{1}$, J. K. O. FERNANDES ${ }^{1,2}$, M. A. F. MARTINS ${ }^{1}$, Y. GUERRIERI ${ }^{1}$ \\ ${ }^{1}$ PEI - Programa de Pós-Graduação em Engenharia Industrial \\ Universidade Federal da Bahia \\ ${ }^{2}$ COPENOR - Companhia Petroquímica do Nordeste. \\ E-mail para contato: yuri.guerrieri@ufba.br
}

\begin{abstract}
RESUMO - Este trabalho resulta de uma solução proposta a um problema industrial de uma planta de produção de hexamina, localizada no Polo Petroquímico de Camaçari. O processo de produção ocorre em um reator tipo CSTR e com reciclo, onde a hexamina é produzida a partir da reação entre formaldeído e amônia. Atualmente, a indicação da taxa de produção média da unidade é conhecida apenas ao final do dia, na apuração da produção total, o que torna ineficiente a gestão da produção da planta. Dentro desse contexto, o objetivo deste trabalho é apresentar uma alternativa para o cálculo da taxa de produção instantânea mediante a modelagem fenomenológica do reator. O modelo proposto é validado com dados de projeto, e face ao seu conhecimento uma ferramenta computacional de interface amigável é desenvolvida no MS EXCEL-VBA, sobre a qual são também avaliadas as incertezas associadas às variáveis de processo através do método de Monte Carlo.
\end{abstract}

\section{INTRODUÇÃO}

A hexamina é um composto branco, cristalino, pouco volátil, solúvel em água e outros solventes, produzida a partir da reação entre formaldeído e amônia. Ela possui ampla aplicação em diversos setores industriais, tais como: indústria de cosméticos; indústria de pneumáticos; agente de cura em resinas fenol-formaldeído; entre outras. O método de produção mais comum de hexamina em escala industrial é a reação do formaldeído com amônia ou sais de amônio na via gás-gás, com os dois componentes na fase gasosa, ou gás-líquido, onde a amônia se encontra na fase gasosa e o formaldeído na fase líquida (Meissner, 1954; Alamdari e Tabkhi, 2004). O resíduo gerado no processo é extremamente resistente à biodegradação (Kaszycki and Koloczek, 2002) sendo assim um sério poluente para recursos hídricos e solos (Taghdiri e Zamani, 2013), o que reforça a relevância em se garantir um processo produtivo eficiente. Neste trabalho, foi feito o acompanhamento numa planta industrial de produção de hexamina onde ficou evidenciado a impossibilidade em se medir a taxa de produção de hexamina após a descarga do reator. Dessa forma, a contabilização da produção só pode ser feita após diversas etapas do processo, o que torna lenta qualquer atuação para melhorias do processo durante a operação. O objetivo deste trabalho é apresentar um modelo rigoroso baseado em balanço de massa e energia para o cálculo da taxa de produção instantânea de hexamina em um reator industrial. A partir desse resultado foi feita uma modelagem rigorosa simplificada considerando a 


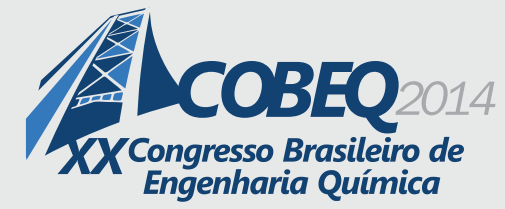

19 a 22 de outubro de 2014

Florianópolis/SC

existência dos medidores da própria planta e avaliada a sua incerteza.

\subsection{Descrição do Processo}

A hexamina é produzida a partir da reação rápida e extremamente exotérmica entre formaldeído e da amônia em temperaturas moderadas (Meissner, 1954). Essa reação química pode ser representada pela Equação 1 válida até uma temperatura de $70^{\circ} \mathrm{C}$. A partir dessa temperatura existe a formação de sub-produtos.

$$
6 \mathrm{H}_{2} \mathrm{CO}_{(a q)}+4 \mathrm{NH}_{3}(a q) \rightarrow\left(\mathrm{CH}_{2}\right)_{6} \mathrm{~N}_{4(a q)}+6 \mathrm{H}_{2} \mathrm{O}_{(l)}
$$

Uma representação simplificada da seção de reação da unidade industrial pode ser vista na Figura 1. A amônia gasosa (corrente 1) e o formaldeído líquido (corrente 2) são alimentados na linha de recirculação (corrente 3 ) do reator, onde circula o produto reacional. Antes de entrar no reator a corrente 3 é resfriada no trocador de calor, onde sua temperatura é controlada e então enviada para o reator através da corrente 4 . A descarga do reator, corrente 5, segue através da bomba e se divide entre a corrente de recirculação e a corrente de produção (corrente 6). Após a seção de reação o produto segue para as seções de evaporação, cristalização, centrifugação, secagem e ensacamento (COPENOR, 2011).

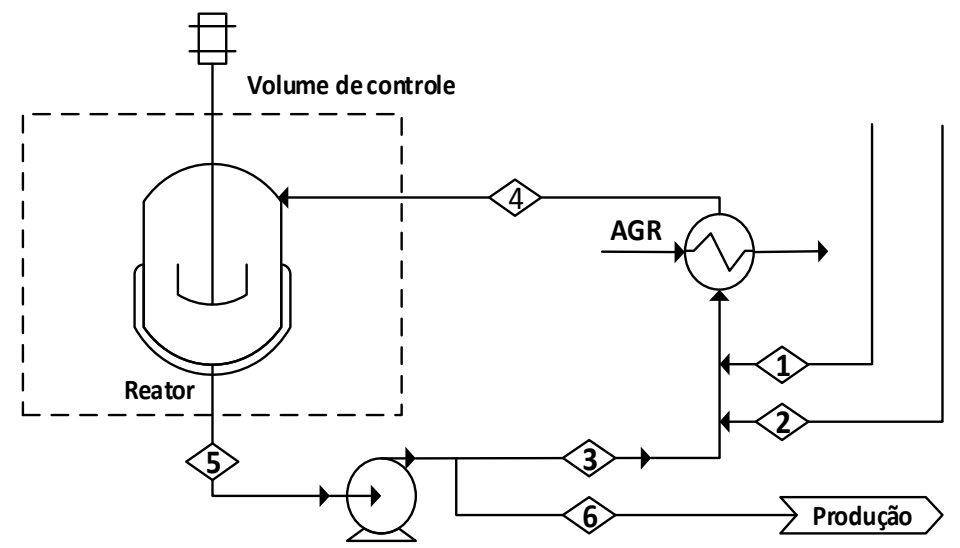

Figura 1 - Fluxograma do sistema de reação, adaptado de MITSUBICHI, S/D.

\section{MODELAGEM RIGOROSA}

Foi desenvolvido um modelo a partir do balanço de massa e energia no reator de hexamina utilizando informações dos manuais da tecnologia, informações encontradas na literatura e algumas hipóteses simplificadoras, que serão apresentadas posteriormente. $\mathrm{O}$ modelo foi feito para o passe no reator e teve um total de 7 variáveis de saída: a temperatura de operação do reator $(T)$, vazão molar para cada componente na descarga do reator $\left(\dot{n}_{i, 5}\right)$ e a vazão mássica de descarga do reator $\dot{m}_{5}$. As entradas do modelo foram arbitradas de acordo com a existência de medidores na planta, conforme 
nomenclatura adotada na Figura 1. São elas: vazão e fração mássica dos componentes da corrente 1, vazão e fração mássica dos componente da corrente 2 , temperatura da corrente 4 e volume reacional.

\subsection{Desenvolvimento do Modelo}

De forma generalizada o balanço de massa por componente no volume de controle definido na Figura 1 pode ser expresso pela Equação 2.

$$
\left(\sum_{j=1}^{2} \dot{n}_{i, j}+\dot{n}_{i, 5} \alpha\right)-\dot{n}_{i, 5}+v_{i} r_{H e x} V=0
$$

Onde:

$\dot{n}_{i, j}$ é a vazão molar do componente $i$ na corrente de entrada $j(\mathrm{kgmol} / \mathrm{h})$

$\dot{m}_{j}$ é a vazão mássica da corrente $j(\mathrm{~kg} / \mathrm{h})$

$x_{i, j}$ é a fração mássica do componente $i$ na corrente $j$ (adimensional)

$V$ é o volume reacional $\left(\mathrm{m}^{3}\right)$

$v_{i}$ é o coeficiente estequiométrico do componente $i$ (adimensional)

$\alpha$ é a razão reciclo/descarga do reator $\left(\alpha=\dot{m}_{3} / \dot{m}_{5}\right)$

$T_{i}$ é a temperatura da corrente $i\left({ }^{\circ} \mathrm{C}\right)$

No modelo foi utilizada a lei de velocidade da reação de formação da hexamina (Equação 3) proposta por Wood e Stevens (1964).

$$
r_{H e x}=1,13 \cdot 10^{9} \exp (-14200 /(R \cdot T)) \cdot C_{A m} \cdot C_{F}{ }^{2}
$$

Os balanços para cada componente estão listados na Tabela 1.

Tabela 1 - Equações dos balanços por componente no reator

\begin{tabular}{|c|c|c|c|}
\hline Componente & Nomenclatura & Equação & \\
\hline Amônia & $A m$ & $\left(\dot{n}_{A m, 1}+\dot{n}_{A m, 5} \alpha\right)-\dot{n}_{A m, 5}-4 \frac{\left(k_{H} \dot{n}_{A m, 5} \dot{n}_{F, 5}{ }^{2}\right) V}{q^{3}}=0$ & $(4)$ \\
\hline Formaldeído & $F$ & $\left(\dot{n}_{F, 2}+\dot{n}_{F, 5} \alpha\right)-\dot{n}_{F, 5}-6 \frac{\left(k_{H} \dot{n}_{A m, 5} \dot{n}_{F, 5}^{2}\right) V}{q^{3}}=0$ & $(5)$ \\
\hline Hexamina & $H e x$ & $\left(0+\dot{n}_{H e x, 5} \alpha\right)-\dot{n}_{H e x, 5}+\frac{\left(k_{H e x} \dot{n}_{A m, 5} \dot{n}_{F, 5}{ }^{2}\right) V}{q^{3}}=0$ & $(6)$ \\
\hline Água & $A g$ & $\left(\dot{n}_{A g, 2}+\dot{n}_{A g, 5} \alpha\right)-\dot{n}_{A g, 5}+6 \frac{\left(k_{H} \dot{n}_{A m, 5} \dot{n}_{F, 5}^{2}\right) V}{q^{3}}=0$ & $(7)$ \\
\hline Metanol & $M$ & $\left(\dot{n}_{M, 2}+\dot{n}_{M, 5} \alpha\right)-\dot{n}_{M, 5}=0$ & $(8)$ \\
\hline
\end{tabular}

$q$ é a vazão volumétrica, definida como $q=\dot{m}_{5} / \rho_{5}$ 
O balanço de massa global no volume de controle é dado pela Equação 9:

$$
\begin{aligned}
& {\left[\left(\dot{n}_{A m, 1}+\dot{n}_{A m, 5} \alpha\right) M M_{A m}+\left(\dot{n}_{F, 2}+\dot{n}_{F, 5} \alpha\right) M M_{F}+\left(0+\dot{n}_{H e x, 5} \alpha\right) M M_{H e x}\right.} \\
& \left.+\left(\dot{n}_{A g, 2}+\dot{n}_{A g, 5} \alpha\right) M M_{A g}+\left(\dot{n}_{M, 2}+\dot{n}_{M, 5} \alpha\right) M M_{M}\right]-\dot{m}_{5}=0
\end{aligned}
$$

Onde $M M_{i}$ é a massa molar do componente $i$.

Considerando o trabalho de eixo desprezível, reator adiabático e calor específico $(C p)$ constante, o balanço de energia no volume de controle é dado pela Equação 10:

$$
\begin{aligned}
-\left[\left(\dot{n}_{A m, 1}+\dot{n}_{A m, 5} \alpha\right) C p_{A m}\left(T-T_{4}\right)+\left(\dot{n}_{F, 2}+\dot{n}_{F, 5} \alpha\right) C p_{F}\left(T-T_{4}\right)\right. \\
+\left(0+\dot{n}_{H, 5} \alpha\right) C p_{H e x}\left(T-T_{4}\right)+\left(\dot{n}_{A g, 2}+\dot{n}_{A g, 5} \alpha\right) C p_{A g}\left(T-T_{4}\right) \\
\left.+\left(\dot{n}_{M, 2}+\dot{n}_{M, 5} \alpha\right) C p_{M}\left(T-T_{4}\right)\right]\left[\Delta H_{H e x}^{o}\right. \\
\left.\quad+\Delta C p\left(T-T_{r e f}\right)\right] \frac{\left(k_{H e x} \dot{n}_{A m, 5} \dot{n}_{F, 5}{ }^{2}\right) V}{q^{3}}=0
\end{aligned}
$$

Por fim, o valor da taxa de produção instantânea na forma de vazão em $\mathrm{kg} / \mathrm{h}$ de hexamina pode ser calculado através da Equação 11.

$$
\dot{m}_{\text {Hinst }}=\dot{n}_{H e x, 5}(1-\alpha) M M_{H e x}
$$

\section{Considerações do Modelo}

Para simplificar o modelo foram feitas algumas considerações. A primeira hipótese admitida é referente ao fato de que apesar de um dos reagentes ser alimentado na fase gasosa, a alimentação se faz em um ejetor e há um trecho longo de tubulação até o reator para garantir a homogeneidade dos reagentes. Assim, assumiu-se que este se dissolve totalmente na mistura líquida de modo que todo o volume reacional encontra-se na fase líquida. Além disso, como a reação deve ser conduzida dentro de uma estreita faixa de temperatura $\left(40^{\circ} \mathrm{C}\right.$ a $\left.70{ }^{\circ} \mathrm{C}\right)$ negligenciou-se a dependência das capacidades caloríficas com a mesma. De forma semelhante, foi negligenciada a dependência da massa específica com a temperatura. Isso pode ser assumido devido à grande concentração de água presente tanto na alimentação quanto no meio reacional e também pela consideração da presença exclusiva de fase líquida no reator. Os valores dos $C p$ e $\rho$ necessários para solução do sistema de equações foram estimados no HYSYS utilizando o modelo termodinâmico NRTL (Carlson, 1996).

\subsection{Implementação do Modelo}

O sistema de equações não-lineares que constituem o modelo é composto pelas Equações 4-7, 9 e 10. A solução deste sistema foi feita através de uma rotina utilizando a função $f$ solve do MATLAB e vazão molar de metanol pode ser calculada através da Equação 12. 


$$
\dot{n}_{M, 5}=\frac{\left[\dot{m}_{5}-\left(\dot{n}_{A m, 5} M M_{A m}+\dot{n}_{F, 5} M M_{F}+\dot{n}_{H e x, 5} M M_{H e x}+\dot{n}_{A g, 5} M M_{A g}\right)\right]}{M M_{M}}
$$

\subsection{Modelo Rigoroso Simplificado}

Apesar de consistente com os dados de projeto da unidade, o modelo obtido poderia ser redefinido de modo que algumas variáveis usadas para definir a condição operacional também fossem entradas para ele. O novo modelo foi feito a partir das equações obtidas inicialmente com o desenvolvimento do modelo do reator manuseadas de forma a incorporar as novas considerações admitidas. Foram elas a temperatura do reator $(T)$ e a vazão mássica de reciclo $\left(\dot{m}_{3}\right)$. Estas, juntamente com as outras variáveis de entrada já mencionadas no modelo rigoroso, e que também são medidas na planta, são as variáveis de entrada deste modelo simplificado. Já as saídas do modelo são: $\dot{n}_{F, 5}$ taxa de formaldeído residual (mol/h), $\dot{n}_{A m, 5}$ - taxa de amônia residual (mol/h), $\dot{m}_{\text {Hex }}$ inst $^{-}$taxa de produção instantânea de hexamina $(\mathrm{kg} / \mathrm{h})$.

Com esta simplificação, a Equação 4 pode ser re-escrita na forma da Equação 13:

$$
\dot{n}_{A m}=\frac{\dot{n}_{A m, 1}}{\left[1-\alpha+4\left(\frac{k_{H e x} \dot{n}_{F}^{2} V}{q^{3}}\right)\right]}
$$

Substituindo a Equação 13 na Equação 5, é possível se escrever a função escrita na Equação 14.

$$
\begin{gathered}
f=\dot{n}_{F}{ }^{3}\left(4 k_{H e x} V \alpha-4 k_{H e x} V\right)+\dot{n}_{F}{ }^{2}\left(4 k_{H} V \dot{n}_{F, 2}-6 k_{H e x} V \dot{n}_{A m, 1}\right) \\
+\dot{n}_{F}\left(2 \alpha q^{3}-\alpha^{2} q^{3}-q^{3}\right)+\dot{n}_{F, 2} q^{3}(1-\alpha)=0
\end{gathered}
$$

Verificou-se que nas condições operacionais avaliadas, o polinômio definido na Equação 14 apresenta duas raízes negativas e uma positiva. Uma vez que o valor de $\dot{n}_{F}$ (vazão molar de formaldeído na descarga), está fisicamente limitado entre zero e a vazão molar de formaldeído na corrente 2 , foi utilizado o método da bisseção para encontrar a única raiz da equação cúbica com real significado físico. A partir daí, o valor de $\dot{n}_{F}$ encontrado pode ser utilizado para a determinação da vazão molar de amônia (Equação 13) e posteriormente usado para o cálculo das vazões molares de hexamina, água e metanol, através das Equações 6, 7 e 12, respectivamente. Com o valor de vazão molar de hexamina, a sua substituição na Equação 11 fornecerá o valor de taxa de produção instantânea na forma de vazão em $\mathrm{kg} / \mathrm{h}$ de hexamina.

\section{RESULTADOS}

A validação do modelo rigoroso foi feita através da comparação do resultado do modelo às condições de projeto. A síntese desse resultado está apresentada na Tabela 2. 
Tabela 2 - Resposta do Modelo Rigoroso

\begin{tabular}{|c|c|c|c|c|}
\hline Variáveis & Unidade & Medido & Modelo & $|\Delta| /(\%)$ \\
\hline$\dot{n}_{A m, 5}$ & $\mathrm{kgmol} / \mathrm{h}$ & 20,55 & 19,92 & 3,082 \\
\hline$\dot{n}_{F, 5}$ & $\mathrm{kgmol} / \mathrm{h}$ & 0 & 0,89 & - \\
\hline$\dot{n}_{H e x, 5}$ & $\mathrm{kgmol} / \mathrm{h}$ & 47,44 & 47,51 & 0,160 \\
\hline$\dot{n}_{A g, 5}$ & $\mathrm{kgmol} / \mathrm{h}$ & 964,47 & 964,15 & 0,033 \\
\hline$\dot{m}_{5}$ & $\mathrm{~kg} / \mathrm{h}$ & 25000 & 25044 & 0,177 \\
\hline $\mathrm{T}$ & ${ }^{\circ} \mathrm{C}$ & 55 & 53,92 & 1,949 \\
\hline$\Delta=100 \cdot($ Medido - Modelo $/$ Medido & & & \\
\hline
\end{tabular}

É possível verificar através dos desvios relativos uma boa aderência entre os dados de projeto (medido) e os valores calculados pelo modelo. Deve-se ressaltar o fato de que o modelo proposto prevê a existência de pequena quantidade de formaldeído residual $\left(\dot{n}_{F, 5}\right)$, embora a condição de projeto não considere a existência deste. Assim, aplicando os valores obtidos na Equação 11, o valor encontrado para taxa de produção instantânea de hexamina $\left(\dot{m}_{H_{\text {inst }}}\right)$ foi de $472,38 \mathrm{~kg} / \mathrm{h}$.

Verificada a capacidade do modelo rigoroso em representar os dados do processo, foi feita uma avaliação do modelo simplificado. Neste caso, além de serem verificados os valores médios calculados pelos modelos, foi avaliada também a sua incerteza. Um dos métodos para o cálculo da incerteza é através do Guia para Expressão da Incerteza de Medição ou GUM (BIPM et al., 2008a), a partir da Equação 15 relativa às contribuições parciais.

$$
u\left(\dot{n}_{B, 5}\right)=\sqrt{\frac{\sum_{i=1}^{N}\left(\frac{\partial f}{\partial x_{i}}\right)^{2} u^{2}\left(x_{i}\right)}{\left(\frac{\partial f}{\partial \dot{n}_{B, 5}}\right)^{2}}}
$$

em que $\mathrm{x}_{\mathrm{i}}$ representa os $N$ parâmetros variáveis de entrada do modelo.

Se a relação funcional entre a grandeza de saída e as grandezas de entrada é não-linear, a expansão de primeira ordem da série de Taylor da relação entre as grandezas pode não ser uma aproximação aceitável (Dietrich, 1991), o que se torna um problema para a aplicabilidade do método GUM. Por outro lado, um método alternativo ao do GUM (GUM S1) para avaliar a incerteza de grandezas físicas é aquele baseado no Método Monte Carlo (MMC) (BIPM et al., 2008b) o qual consiste em avaliar as incertezas de grandezas de saída de um modelo (linear ou não-linear) a partir da propagação de PDFs das grandezas de entrada, construindo assim as distribuições de probabilidade empíricas das grandezas de saída. Foi considerado que todas as grandezas de entrada são não correlacionadas e exibem PDFs normais. Uma vez conhecidas as PDFs das grandezas de saída, podem-se estimar quaisquer momentos estatísticos, tais como, primeiro momento (média), segundo momento (desvio-padrão ou incerteza), bem como os intervalos de abrangência para uma dada probabilidade. 


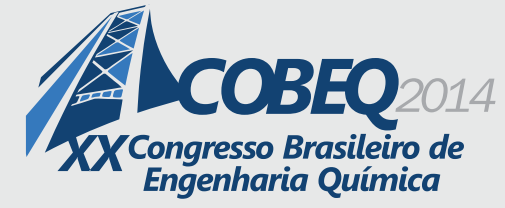

Como a relação entre as grandezas de saída e a grandezas de entrada para o modelo é não linear, optou-se pelo uso do MMC para a avaliação da incerteza das grandezas de saída do modelo simplificado. A incerteza de todas as variáveis de entrada foi arbitrada em $1 \%$ dos valores médios dessas grandezas e foram utilizados $10^{6}$ pontos para a simulação do MMC. As PDFs empíricas das grandezas de saída estão apresentadas na Figura 2, ao passo que seus valores de média, incerteza padrão e os intervalos de abrangência, para uma probabilidade de $68 \%$, encontram-se na Tabela 3 . As linhas que podem ser vistas nos gráficos da Figura 2 delimitam os intervalos superior e inferior considerando 1 desvio padrão. Comparando os valores médios obtidos por ambos os modelos, observa-se que o modelo simplificado gerou resultados semelhantes aos gerados pelo modelo rigoroso.

Tabela 3 - Resposta do Modelo Rigoroso Simplificado - Simulação pelo MMC

\begin{tabular}{|c|c|c|c|c|c|}
\hline & Unidades & Média & Incerteza padrão & Limite inferior & Limite superior \\
\hline$\dot{n}_{A m, 5}$ & $\mathrm{kgmol} / \mathrm{h}$ & 20 & 4 & 16 & 24 \\
\hline$\dot{n}_{F, 5}$ & $\mathrm{kgmol} / \mathrm{h}$ & 0,87 & 0,10 & 0,77 & 0,97 \\
\hline$\dot{m}_{H_{\text {inst }}}$ & $\mathrm{kg} / \mathrm{h}$ & 473 & 7 & 466 & 480 \\
\hline
\end{tabular}

Embora as incertezas da amônia e do formaldeído residual estimadas pelo MMC sejam significativas os seus valores são de ordem de grandeza compatíveis com a das variáveis avaliadas. Por outro lado, a incerteza dessas variáveis e da taxa de hexamina estimada pelo GUM apresentou resultados incoerentes, o que pode ser uma consequência de grande não-linearidade do modelo na condição avaliada.

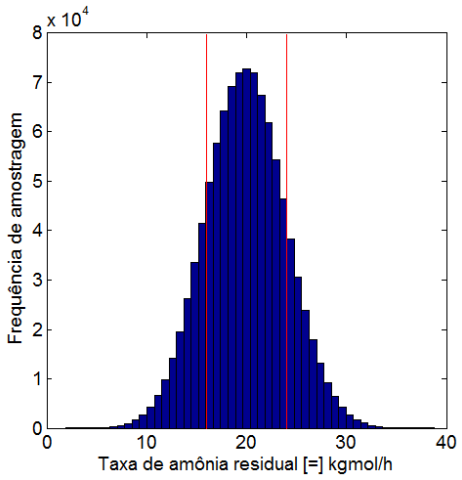

(a)

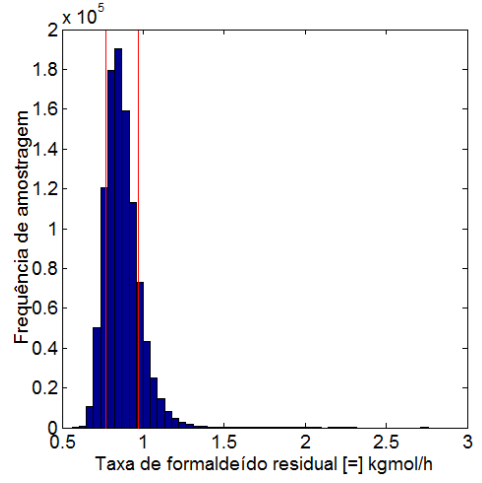

(b)

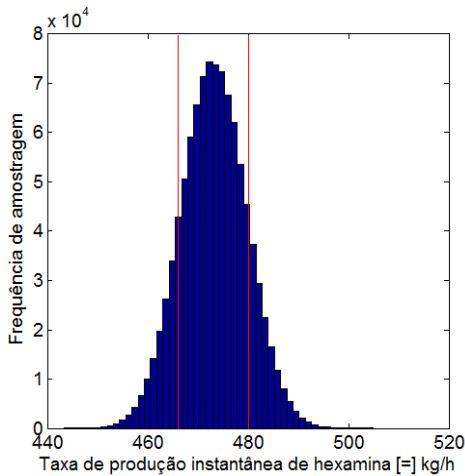

(c)

Figura 2 - PDFs das grandezas de saída: a) taxa de amônia residual, b) taxa de formaldeído resitual e c) taxa de produção instantânea de hexamina.

\section{CONCLUSÃO}

O estudo em busca de soluções para um problema industrial real em uma planta de Hexamina levou ao desenvolvimento de dois modelos em estado estacionário do reator principal do processo. $\mathrm{O}$ modelo obtido através da modelagem rigorosa foi confrontado com dados de fluxograma de processo 


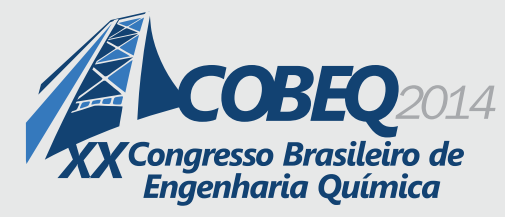

e obteve uma discrepância máxima de 3,18\%. Já o modelo simplificado apresentou resultados coerentes e próximos dos valores obtidos pelo modelo rigoroso, mostrando-se também válido para representar o processo. A utlização do modelo simplificado mostrou-se bastate vantajoso uma vez que teve boa qualidade no resultado e possui solução numérica simples e convergência garantida. A incerteza deste modelo foi analisada via GUM e MMC, onde o MMC mostrou-se mais adequada e detectou-se a necessidade do uso do MMC já que a relação funcional entre a saída e as grandezas de entrada é não-linear.

\section{REFERÊNCIAS}

ALAMDARI, A; TABKHI, F. Kinetics of hexamine crystallization in industrial scale. Chem Eng Process, p. 803-810, 2004

BIPM, IEC, IFCC, ILAC, ISO, IUPAC, IUPAP and OIML. Evaluation of Measurement Data - Guide to the Expression of Uncertainty in Measurement (GUM 1995 with minor corrections), Joint Committee for Guides in Metrology, JCGM 100, 2008 a.

BIPM, IEC, IFCC, ILAC, ISO, IUPAC, IUPAP and OIML. Evaluation of Measurement DataSupplement 1 to the Guide to the Expression of Uncertainty in Measurement - Propagation of distributions using a Monte Carlo method, Joint Committee for Guides in Metrology, JCGM $101,2008 b$.

CARLSON, E. Don't gamble with physical properties for simulations. Chemical Engineering Progress, Cambridge, p. 35-46, 1996.

COPENOR. Descrição de Processo da Unidade de Hexametilenotetramina. COPENOR. 2011.

DIETRICH, C. F., Uncertainty, calibration and probability, 2nd ed., Adam-Hilger, 1991.

KASZYCKI, P; KOLOCZEK, H. Biodegradation of formaldehyde and its derivatives in industrial wastewater with methylotrophic yeast Hansenula polymorpha and with yeast-augmented activated sludge. Biodegradation, v. 13, p. 91-99, 2002.

MEISSNER, F. Continuous Production of Hemathylenetetramin. Industrial and Engineering Chemistry, Cologne, v. 46, p. 724-727, 1954.

MITSUBISHI., K. K. L. Hexamine Plant Material \& Heat Balance Sheet, COPENOR. Tokyo. S/D.

TAGHDIRI, M; ZAMANI, N. Hexamine adsorption study on activated carbon from aqueous solutions for application in treatment of hexamine industrial wastewater. Int. J. Environ. Sci. Technol. v. 10, 19-26, 2013.

WOOD, R. K.; STEVENS, W. F. Reaction Kinetics of the Formation of Hexamethylenetetramin. Journal of Applied Chemistry, Ottawa, p. 325-330, 1964. 